Commun. Korean Math. Soc. 28 (2013), No. 4, pp. 649-667

http://dx.doi.org/10.4134/CKMS.2013.28.4.649

\title{
AN EXPLICIT FORMULA FOR THE NUMBER OF SUBGROUPS OF A FINITE ABELIAN $p$-GROUP UP TO RANK 3
}

\author{
Ju-MoK OH
}

\begin{abstract}
In this paper we give an explicit formula for the total number of subgroups of a finite abelian $p$-group up to rank three.
\end{abstract}

\section{Introduction}

Given a finite abelian group what is the total number of subgroups? This problem can be reduced to that of finding the number of subgroups of a finite abelian $p$-group because every finite abelian group is the direct product of its Sylow subgroups. Several different versions of the formula for the number of certain type subgroups of a given finite abelian $p$-group have been known (for example see $[2,3,4,6])$. But in general these formulas do not lead us to an explicit formula for the total number of subgroups, which is well explained in [1]. As a result of this direction, G. Călugăreanu [1] and later J. Petrillo [5] have given an explicit formula for the total number of subgroups of a finite abelian $p$-group of rank two by using Goursat's Theorem. In this paper we reprove their result by finding its recurrence relation and as a new result we give an explicit formula for the total number of subgroups of a finite abelian p-group of rank three by a similar method.

\section{The total number of subgroups of a finite abelian $p$-group up to rank 3}

The following is the main result of this paper, which will be proved in the next section.

Theorem 2.1. Let

$$
\mathbb{Z}_{p^{m}} \times \mathbb{Z}_{p^{n}} \times \mathbb{Z}_{p^{\ell}}=\left\langle a, b, c \mid a^{p^{m}}=b^{p^{n}}=c^{p^{\ell}}=e,[a, b]=[a, c]=[b, c]=e\right\rangle
$$

Received October 7, 2011.

2010 Mathematics Subject Classification. Primary 05A15, 20K01, 20 K27.

Key words and phrases. enumeration, subgroup, abelian $p$-group.

This work was supported by the Research Institute of Natural Science of GangneungWonju National University. 
be an abelian $p$-group of order $p^{m+n+\ell}$ where $m, n$ and $\ell$ are non-negative integers such that $m \geq n \geq \ell$ and $p$ is a prime number. Then the total number $S\left(\mathbb{Z}_{p^{m}} \times \mathbb{Z}_{p^{n}} \times \mathbb{Z}_{p^{\ell}}\right)$ of subgroups of $\mathbb{Z}_{p^{m}} \times \mathbb{Z}_{p^{n}} \times \mathbb{Z}_{p^{\ell}}$ is

$$
S\left(\mathbb{Z}_{p^{m}} \times \mathbb{Z}_{p^{n}} \times \mathbb{Z}_{p^{\ell}}\right)=\sum_{t=1}^{\ell} t\left[(m+n+\ell-3 t+4) p^{2(t-1)}\right.
$$

$$
\begin{array}{r}
\left.+(m+n+\ell-3 t+2) p^{2(t-1)+1}\right] \\
+\sum_{k=\ell}^{n}(\ell+1)(m+n+1-2 k) p^{k+\ell}
\end{array}
$$

where the first iterated sum is 0 when $\ell=0$.

We now evaluate Eq. (1) more specifically. Since

$$
\begin{aligned}
\left(p^{2}-1\right) \sum_{t=1}^{\ell} t p^{2 t} & =p^{2} \sum_{t=1}^{\ell} t p^{2 t}-\sum_{t=1}^{\ell} t p^{2 t} \\
& =\left(\ell p^{2 \ell+2}+p^{2} \sum_{t=1}^{\ell-1} t p^{2 t}\right)-\left(p^{2}+p^{2} \sum_{t=1}^{\ell-1}(t+1) p^{2 t}\right) \\
& =\ell p^{2 \ell+2}-p^{2} \sum_{t=0}^{\ell-1} p^{2 t}=\ell p^{2 \ell+2}-p^{2} \frac{p^{2 \ell}-1}{p^{2}-1},
\end{aligned}
$$

we have

$$
\sum_{t=1}^{\ell} t p^{2 t}=\frac{\ell p^{2 \ell+2}}{p^{2}-1}-p^{2} \frac{p^{2 \ell}-1}{\left(p^{2}-1\right)^{2}}
$$

Since

$$
\begin{aligned}
& \left(p^{2}-1\right) \sum_{t=1}^{\ell} t^{2} p^{2 t} \\
= & p^{2} \sum_{t=1}^{\ell} t^{2} p^{2 t}-\sum_{t=1}^{\ell} t^{2} p^{2 t} \\
= & \left(\ell^{2} p^{2 \ell+2}+p^{2} \sum_{t=1}^{\ell-1} t^{2} p^{2 t}\right)-\left(p^{2}+p^{2} \sum_{t=1}^{\ell-1}(t+1)^{2} p^{2 t}\right) \\
= & \ell^{2} p^{2 \ell+2}-p^{2}+p^{2} \sum_{t=1}^{\ell-1}(-2 t-1) p^{2 t} \\
= & \ell^{2} p^{2 \ell+2}-p^{2} \sum_{t=0}^{\ell-1} p^{2 t}-2 p^{2} \sum_{t=1}^{\ell-1} t p^{2 t}
\end{aligned}
$$




$$
=\ell^{2} p^{2 \ell+2}-p^{2} \frac{p^{2 \ell}-1}{p^{2}-1}-2 p^{2}\left[\frac{(\ell-1) p^{2 \ell}}{p^{2}-1}-p^{2} \frac{p^{2 \ell-2}-1}{\left(p^{2}-1\right)^{2}}\right] \text { by Eq. (2), }
$$

we have

$$
\text { (3) } \sum_{t=1}^{\ell} t^{2} p^{2 t}=\frac{\ell^{2} p^{2 \ell+2}}{p^{2}-1}-p^{2} \frac{p^{2 \ell}-1}{\left(p^{2}-1\right)^{2}}-2 p^{2}\left[\frac{(\ell-1) p^{2 \ell}}{\left(p^{2}-1\right)^{2}}-p^{2} \frac{p^{2 \ell-2}-1}{\left(p^{2}-1\right)^{3}}\right] \text {. }
$$

Since

$$
\begin{aligned}
(p-1) \sum_{k=1}^{n} k p^{k} & =p \sum_{k=1}^{n} k p^{k}-\sum_{k=1}^{n} k p^{k} \\
& =\left(n p^{n+1}+p \sum_{k=1}^{n-1} k p^{k}\right)-\left(p+p \sum_{k=1}^{n-1}(k+1) p^{k}\right) \\
& =n p^{n+1}-p \sum_{k=0}^{n-1} p^{k}=n p^{n+1}-p \frac{p^{n}-1}{p-1},
\end{aligned}
$$

we have

(4)

$$
\sum_{k=1}^{n} k p^{k}=\frac{n p^{n+1}}{p-1}-p \frac{p^{n}-1}{(p-1)^{2}}
$$

Using Eqs. (2)-(4) we get that

$$
\begin{aligned}
& \sum_{t=1}^{\ell} t\left[(m+n+\ell-3 t+4) p^{2(t-1)}+(m+n+\ell-3 t+2) p^{2(t-1)+1}\right] \\
& +\sum_{k=\ell}^{n}(\ell+1)(m+n+1-2 k) p^{k+\ell} \\
= & \sum_{t=1}^{\ell} t\left[(m+n+\ell-3 t+4) p^{2(t-1)}+(m+n+\ell-3 t+2) p^{2(t-1)+1}\right] \\
& +\sum_{k=0}^{n}(\ell+1)(m+n+1-2 k) p^{k+\ell}-\sum_{k=0}^{\ell-1}(\ell+1)(m+n+1-2 k) p^{k+\ell} \\
= & {\left[\frac{m+n+\ell+4}{p^{2}}+\frac{m+n+\ell+2}{p}\right] \sum_{t=1}^{\ell} t p^{2 t}-3\left[\frac{1}{p^{2}}+\frac{1}{p}\right] \sum_{t=1}^{\ell} t^{2} p^{2 t} } \\
& +(\ell+1)(m+n+1) p^{\ell}\left[\sum_{k=0}^{n} p^{k}-\sum_{k=0}^{\ell-1} p^{k}\right]-2(\ell+1) p^{\ell}\left[\sum_{k=0}^{n} k p^{k}-\sum_{k=0}^{\ell-1} k p^{k}\right] \\
= & \frac{(m+n-2 \ell+4) \ell p^{2 \ell}+(m+n-2 \ell+2) \ell p^{2 \ell+1}}{p^{2}-1} \\
& -\frac{(m+n-5 \ell+7) p^{2 \ell}+(m+n-5 \ell+5) p^{2 \ell+1}-(m+n+\ell-1) p-m-n-\ell-1}{\left(p^{2}-1\right)^{2}}
\end{aligned}
$$




$$
\begin{aligned}
& -\frac{6\left(p^{2 \ell}+p^{2 \ell+1}-p^{3}-p^{2}\right)}{\left(p^{2}-1\right)^{3}}+\frac{(\ell+1)\left((m-n+1) p^{n+\ell+1}-(m+n-2 \ell+3) p^{2 \ell}\right)}{p-1} \\
& +\frac{2(\ell+1)\left(p^{n+\ell+1}-p^{2 \ell}\right)}{(p-1)^{2}} .
\end{aligned}
$$

Therefore, we have proved the following.

Corollary 2.2. Let

$$
\mathbb{Z}_{p^{m}} \times \mathbb{Z}_{p^{n}} \times \mathbb{Z}_{p^{\ell}}=\left\langle a, b, c \mid a^{p^{m}}=b^{p^{n}}=c^{p^{\ell}}=e,[a, b]=[a, c]=[b, c]=e\right\rangle
$$

be an abelian $p$-group of order $p^{m+n+\ell}$ where $m, n$ and $\ell$ are non-negative integers such that $m \geq n \geq \ell$ and $p$ is a prime number. Then the total number $S\left(\mathbb{Z}_{p^{m}} \times \mathbb{Z}_{p^{n}} \times \mathbb{Z}_{p^{\ell}}\right)$ of subgroups of $\mathbb{Z}_{p^{m}} \times \mathbb{Z}_{p^{n}} \times \mathbb{Z}_{p^{\ell}}$ is

$$
\begin{aligned}
& S\left(\mathbb{Z}_{p^{m}} \times \mathbb{Z}_{p^{n}} \times \mathbb{Z}_{p^{\ell}}\right)=\frac{(m+n-2 \ell+4) \ell p^{2 \ell}+(m+n-2 \ell+2) \ell p^{2 \ell+1}}{p^{2}-1} \\
& -\frac{(m+n-5 \ell+7) p^{2 \ell}+(m+n-5 \ell+5) p^{2 \ell+1}-(m+n+\ell-1) p-m-n-\ell-1}{\left(p^{2}-1\right)^{2}} \\
& -\frac{6\left(p^{2 \ell}+p^{2 \ell+1}-p^{3}-p^{2}\right)}{\left(p^{2}-1\right)^{3}}+\frac{(\ell+1)\left((m-n+1) p^{n+\ell+1}-(m+n-2 \ell+3) p^{2 \ell}\right)}{p-1} \\
& +\frac{2(\ell+1)\left(p^{n+\ell+1}-p^{2 \ell}\right)}{(p-1)^{2}} .
\end{aligned}
$$

\section{The proof of Theorem 2.1}

Given a finite group $G$ let $\mathcal{S}(G)$ and $\mathcal{T}(G)$ be the set of subgroups of $G$ and the set of proper subgroups of $G$, respectively. Let $S(G):=|\mathcal{S}(G)|$ and $T(G):=|\mathcal{T}(G)|$.

Throughout the section we assume that

$$
\mathbb{Z}_{p^{m}} \times \mathbb{Z}_{p^{n}} \times \mathbb{Z}_{p^{\ell}}=\left\langle a, b, c \mid a^{p^{m}}=b^{p^{n}}=c^{p^{\ell}}=e,[a, b]=[a, c]=[b, c]=e\right\rangle
$$

is an abelian $p$-group of order $p^{m+n+\ell}$ where $m, n$ and $\ell$ are non-negative integers such that $m \geq n \geq \ell$ and $p$ is a prime number. Let

$$
b_{m, n, \ell}:=S\left(\mathbb{Z}_{p^{m}} \times \mathbb{Z}_{p^{n}} \times \mathbb{Z}_{p^{\ell}}\right),
$$

where $m \geq n \geq \ell$. For convenience of notation we set $b_{m, n}:=b_{m, n, 0}$ and $b_{m}:=b_{m, 0,0}$

Clearly $b_{m}=S\left(\mathbb{Z}_{p^{m}}\right)=m+1$. In the following we consider the case for rank 2 and 3 separately.

\subsection{The number of subgroups of $\mathbb{Z}_{p^{m}} \times \mathbb{Z}_{p^{n}}$}

In elementary group theory, the following is well-known.

Lemma 3.1. Assume that $m$ and $n$ are positive integers. The group $\mathbb{Z}_{p^{m}} \times \mathbb{Z}_{p^{n}}$ has $(p+1)$ index p subgroups $\left\langle a^{p}, b\right\rangle \cong \mathbb{Z}_{p^{m-1}} \times \mathbb{Z}_{p^{n}},\left\langle b^{p}, a^{i} b\right\rangle \cong \mathbb{Z}_{p^{m}} \times \mathbb{Z}_{p^{n-1}}$, $i=1,2, \ldots, p-1$, and $\left\langle a, b^{p}\right\rangle \cong \mathbb{Z}_{p^{m}} \times \mathbb{Z}_{p^{n-1}}$. 
Lemma 3.2. (1) If $m>n$, then

$$
b_{m, n}=b_{m-1, n}+p\left(b_{m, n-1}-b_{m-1, n-1}\right)+1 .
$$

(2) If $m=n$, then

$$
b_{m, m}=(p+1) b_{m, m-1}-p b_{m-1, m-1}+1 .
$$

Proof. We only give the proof when $m>n$. The remaining can be proved similarly.

By Lemma 3.1 we have

$$
\begin{aligned}
\mathcal{T}\left(\mathbb{Z}_{p^{m}} \times \mathbb{Z}_{p^{n}}\right)=\mathcal{S}\left(\left\langle a^{p}, b\right\rangle \cong \mathbb{Z}_{p^{m-1}} \times \mathbb{Z}_{p^{n}}\right) & \bigcup_{i=1}^{p-1} \mathcal{S}\left(\left\langle b^{p}, a^{i} b\right\rangle \cong \mathbb{Z}_{p^{m}} \times \mathbb{Z}_{p^{n-1}}\right) \\
& \bigcup \mathcal{S}\left(\left\langle a, b^{p}\right\rangle \cong \mathbb{Z}_{p^{m}} \times \mathbb{Z}_{p^{n-1}}\right) .
\end{aligned}
$$

Using the inclusion-exclusion principle we have

$$
\begin{aligned}
b_{m, n}-1= & b_{m-1, n}+p b_{m, n-1}-\left(\begin{array}{c}
p+1 \\
2
\end{array}\right) b_{m-1, n-1}+\left(\begin{array}{c}
p+1 \\
3
\end{array}\right) b_{m-1, n-1} \\
& +\cdots+(-1)^{p+2}\left(\begin{array}{c}
p+1 \\
p+1
\end{array}\right) b_{m-1, n-1} \\
= & b_{m-1, n}+p b_{m, n-1}-p b_{m-1, n-1} .
\end{aligned}
$$

Thus

$$
b_{m, n}=b_{m-1, n}+p\left(b_{m, n-1}-b_{m-1, n-1}\right)+1 .
$$

As commented in Introduction, the following is already proved in $[1,5]$. We reprove it by using Lemma 3.2.

\section{Lemma 3.3.}

$$
b_{m, n}=\sum_{k=0}^{n}(m+n+1-2 k) p^{k} .
$$

Proof. We prove Eq. (7) by induction on $n$. Assume first that $n=1$. Since $b_{m, 0}=S\left(\mathbb{Z}_{p^{m}}\right)=m+1$ and $b_{0,0}=S(\langle e\rangle)=1$, Eq. (5) with $n=1$ gives us that

$$
b_{m, 1}=b_{m-1,1}+p+1 \text {. }
$$

Thus

$$
b_{m, 1}=b_{1,1}+(m-1)(p+1) .
$$

Since $b_{1,1}=p+3$ by Eq. (6) with $m=1$, we have

$$
b_{m, 1}=p+3+(m-1)(p+1) .
$$

Hence Eq. (7) holds for $n=1$.

Assume now that Eq. (7) holds from 1 to $n$ and consider the case for $n+1$. By Eq. (5) replacing $n$ by $n+1$ we have

$$
b_{m, n+1}=b_{m-1, n+1}+p\left(b_{m, n}-b_{m-1, n}\right)+1 .
$$


Since

$$
\begin{aligned}
p\left(b_{m, n}-b_{m-1, n}\right) & =p\left[\sum_{k=0}^{n}(m+n+1-2 k) p^{k}-\sum_{k=0}^{n}(m-1+n+1-2 k) p^{k}\right] \\
& =\sum_{k=0}^{n} p^{k+1}
\end{aligned}
$$

by induction hypothesis, we have

$$
b_{m, n+1}=b_{m-1, n+1}+\sum_{k=0}^{n} p^{k+1}+1=b_{m-1, n+1}+\sum_{k=0}^{n+1} p^{k},
$$

which implies that

$$
b_{m, n+1}=b_{n+1, n+1}+(m-n-1) \sum_{k=0}^{n+1} p^{k} .
$$

Furthermore, since

$$
\begin{aligned}
b_{n+1, n+1} & =(p+1) b_{n+1, n}-p b_{n, n}+1 \\
& =(p+1) \sum_{k=0}^{n}(n+1+n+1-2 k) p^{k}-p \sum_{k=0}^{n}(n+n+1-2 k) p^{k}+1 \\
& =\sum_{k=0}^{n+1}(2 n+3-2 k) p^{k}
\end{aligned}
$$

by induction hypothesis, we have

$$
b_{m, n+1}=\sum_{k=0}^{n+1}(2 n+3-2 k) p^{k}+(m-n-1) \sum_{k=0}^{n+1} p^{k}=\sum_{k=0}^{n+1}(m+n+2-2 k) p^{k} .
$$

Hence Eq. (7) holds for $n+1$.

\subsection{The number of subgroups of $\mathbb{Z}_{p^{m}} \times \mathbb{Z}_{p^{n}} \times \mathbb{Z}_{p^{\ell}}$}

Given a positive integer $n$ let $\mathbb{Z}_{n}$ be the cyclic group of order $n$ with the addictive operation. By $\mathbb{Z}_{n}^{*}$ we denote the multiplicative group, that is, the group consisting of all multiplicatively invertible elements of $\mathbb{Z}_{n}$.

In elementary group theory, the following is well-known.

Lemma 3.4. Assume that $m, n$ and $\ell$ are positive integers. The group $\mathbb{Z}_{p^{m}} \times$ $\mathbb{Z}_{p^{n}} \times \mathbb{Z}_{p^{e}}$ has $\left(p^{2}+p+1\right)$ index $p$ subgroups as follows.

(1) $\left\langle a, b, c^{p}\right\rangle \cong \mathbb{Z}_{p^{m}} \times \mathbb{Z}_{p^{n}} \times \mathbb{Z}_{p^{\ell-1}}$,

(2) $\left\langle a^{i} b, b^{p}, c\right\rangle \cong \mathbb{Z}_{p^{m}} \times \mathbb{Z}_{p^{n-1}} \times \mathbb{Z}_{p^{\ell}} ; i=1,2, \ldots, p-1$,

(3) $\left\langle a^{i} b, b^{j} c, c^{p}\right\rangle \cong \mathbb{Z}_{p^{m}} \times \mathbb{Z}_{p^{n}} \times \mathbb{Z}_{p^{\ell-1}} ; i=1,2, \ldots, p-1$ and $j=1,2, \ldots$, $p-1$,

(4) $\left\langle a^{p}, b, c\right\rangle \cong \mathbb{Z}_{p^{m-1}} \times \mathbb{Z}_{p^{n}} \times \mathbb{Z}_{p^{\ell}}$,

(5) $\left\langle a^{i} c, b, c^{p}\right\rangle \cong \mathbb{Z}_{p^{m}} \times \mathbb{Z}_{p^{n}} \times \mathbb{Z}_{p^{\ell-1}} ; i=1,2, \ldots, p-1$, 
(6) $\left\langle a, b^{p}, c\right\rangle \cong \mathbb{Z}_{p^{m}} \times \mathbb{Z}_{p^{n-1}} \times \mathbb{Z}_{p^{\ell}}$,

(7) $\left\langle a, b^{i} c, c^{p}\right\rangle \cong \mathbb{Z}_{p^{m}} \times \mathbb{Z}_{p^{n}} \times \mathbb{Z}_{p^{\ell-1}} ; i=1,2, \ldots, p-1$.

Note that every index $p$ subgroup of $\mathbb{Z}_{p^{m}} \times \mathbb{Z}_{p^{n}} \times \mathbb{Z}_{p^{\ell}}$ contains the subgroup $\left\langle a^{p}, b^{p}, c^{p}\right\rangle \cong \mathbb{Z}_{p^{m-1}} \times \mathbb{Z}_{p^{n-1}} \times \mathbb{Z}_{p^{\ell-1}}$. In the next lemma we find all index $p^{2}$ subgroups of $\mathbb{Z}_{p^{m}} \times \mathbb{Z}_{p^{n}} \times \mathbb{Z}_{p^{\ell}}$ containing $\left\langle a^{p}, b^{p}, c^{p}\right\rangle$.

Lemma 3.5. Assume that $m, n$ and $\ell$ are positive integers. There exist $\left(p^{2}+p+\right.$ 1) index $p^{2}$ subgroups of $\mathbb{Z}_{p^{m}} \times \mathbb{Z}_{p^{n}} \times \mathbb{Z}_{p^{e}}$ containing the subgroup $\left\langle a^{p}, b^{p}, c^{p}\right\rangle \cong$ $\mathbb{Z}_{p^{m-1}} \times \mathbb{Z}_{p^{n-1}} \times \mathbb{Z}_{p^{\ell-1}}$ as follows.

(1) $\left\langle a^{i} b^{j} c, b^{p}, c^{p}\right\rangle \cong \mathbb{Z}_{p^{m}} \times \mathbb{Z}_{p^{n-1}} \times \mathbb{Z}_{p^{\ell-1}} ; i=1,2, \ldots, p-1$ and $j=$ $0,1, \ldots, p-1$,

(2) $\left\langle a^{p}, b^{k} c, c^{p}\right\rangle \cong \mathbb{Z}_{p^{m-1}} \times \mathbb{Z}_{p^{n}} \times \mathbb{Z}_{p^{\ell-1}} ; k=1,2, \ldots, p-1$,

(3) $\left\langle a^{p}, b^{p}, c\right\rangle \cong \mathbb{Z}_{p^{m-1}} \times \mathbb{Z}_{p^{n-1}} \times \mathbb{Z}_{p^{\ell}}$,

(4) $\left\langle a^{i} b, b^{p}, c^{p}\right\rangle \cong \mathbb{Z}_{p^{m}} \times \mathbb{Z}_{p^{n-1}} \times \mathbb{Z}_{p^{\ell-1}} ; i=1,2, \ldots, p-1$,

(5) $\left\langle a^{p}, b, c^{p}\right\rangle \cong \mathbb{Z}_{p^{m-1}} \times \mathbb{Z}_{p^{n}} \times \mathbb{Z}_{p^{\ell-1}}$,

(6) $\left\langle a, b^{p}, c^{p}\right\rangle \cong \mathbb{Z}_{p^{m}} \times \mathbb{Z}_{p^{n-1}} \times \mathbb{Z}_{p^{\ell-1}}$.

Proof. Let $K$ be an index $p^{2}$ subgroup of $\mathbb{Z}_{p^{m}} \times \mathbb{Z}_{p^{n}} \times \mathbb{Z}_{p^{\ell}}$ containing the subgroup $\left\langle a^{p}, b^{p}, c^{p}\right\rangle$. Then we have $a^{i} b^{j} c^{k} \in K$ for some integers $i, j$ and $k$ such that $0 \leq i, j, k \leq p-1$ and $(i, j, k) \neq(0,0,0)$. We now divide the argument into two cases depending on $i=0$ or not.

Case 1: $i \neq 0$. If $j=k=0$, then $K \geq\left\langle a^{p}, b^{p}, c^{p}, a^{i}\right\rangle=\left\langle a, b^{p}, c^{p}\right\rangle$, and hence $K=\left\langle a, b^{p}, c^{p}\right\rangle \cong \mathbb{Z}_{p^{m}} \times \mathbb{Z}_{p^{n-1}} \times \mathbb{Z}_{p^{\ell-1}}$. If $j \neq 0$ and $k=0$, then $K \geq\left\langle a^{p}, b^{p}, c^{p}, a^{i} b^{j}\right\rangle$. Since $j \in \mathbb{Z}_{p^{n}}^{*}$, there exists $j^{\prime} \in \mathbb{Z}_{p^{n}}^{*}$ such that $j j^{\prime} \equiv 1$ $\left(\bmod p^{n}\right)$. So $K \geq\left\langle a^{p}, b^{p}, c^{p}, a^{i} b^{j}\right\rangle=\left\langle a^{p}, b^{p}, c^{p},\left(a^{i} b^{j}\right)^{j^{\prime}}=a^{i j^{\prime}} b\right\rangle$, and hence $K=\left\langle a^{i j^{\prime}} b, b^{p}, c^{p}\right\rangle \cong \mathbb{Z}_{p^{m}} \times \mathbb{Z}_{p^{n-1}} \times \mathbb{Z}_{p^{\ell-1}}$. If $j=0$ and $k \neq 0$, then $K \geq$ $\left\langle a^{p}, b^{p}, c^{p}, a^{i} c^{k}\right\rangle$. Since $k \in \mathbb{Z}_{p^{e}}^{*}$, there exists $k^{\prime} \in \mathbb{Z}_{p^{\ell}}^{*}$ such that $k k^{\prime} \equiv 1$ $\left(\bmod p^{\ell}\right)$. So $K \geq\left\langle a^{p}, b^{p}, c^{p}, a^{i} c^{k}\right\rangle=\left\langle a^{p}, b^{p}, c^{p},\left(a^{i} c^{k}\right)^{k^{\prime}}=a^{i k^{\prime}} c\right\rangle$, and hence $K=\left\langle a^{i k^{\prime}} c, b^{p}, c^{p}\right\rangle \cong \mathbb{Z}_{p^{m}} \times \mathbb{Z}_{p^{n-1}} \times \mathbb{Z}_{p^{\ell-1}}$. If $j \neq 0$ and $k \neq 0$, then $K \geq$ $\left\langle a^{p}, b^{p}, c^{p}, a^{i} b^{j} c^{k}\right\rangle$. Since $k \in \mathbb{Z}_{p^{\ell}}^{*}$, there exists $k^{\prime} \in \mathbb{Z}_{p^{\ell}}^{*}$ such that $k k^{\prime} \equiv 1$ $\left(\bmod p^{\ell}\right)$. So $K \geq\left\langle a^{p}, b^{p}, c^{p}, a^{i} b^{j} c^{k}\right\rangle=\left\langle a^{p}, b^{p}, c^{p},\left(a^{i} b^{j} c^{k}\right)^{k^{\prime}}=a^{i k^{\prime}} b^{j k^{\prime}} c\right\rangle$, and hence $K=\left\langle a^{i k^{\prime}} b^{j k^{\prime}} c, b^{p}, c^{p}\right\rangle \cong \mathbb{Z}_{p^{m}} \times \mathbb{Z}_{p^{n-1}} \times \mathbb{Z}_{p^{\ell-1}}$.

Case 2: $i=0$. If $j=0$, then $k \neq 0$ and $K \geq\left\langle a^{p}, b^{p}, c^{p}, c^{k}\right\rangle=\left\langle a^{p}, b^{p}, c\right\rangle$, and hence $K=\left\langle a^{p}, b^{p}, c\right\rangle \cong \mathbb{Z}_{p^{m-1}} \times \mathbb{Z}_{p^{n-1}} \times \mathbb{Z}_{p^{\ell}}$. If $j \neq 0$ and $k=0$, then $K \geq\left\langle a^{p}, b^{p}, c^{p}, b^{j}\right\rangle=\left\langle a^{p}, b, c^{p}\right\rangle$, and hence $K=\left\langle a^{p}, b, c^{p}\right\rangle \cong \mathbb{Z}_{p^{m-1}} \times$ $\mathbb{Z}_{p^{n}} \times \mathbb{Z}_{p^{\ell-1}}$. If $j \neq 0$ and $k \neq 0$, then $K \geq\left\langle a^{p}, b^{p}, c^{p}, b^{j} c^{k}\right\rangle$. Since $k \in \mathbb{Z}_{p^{\ell}}^{*}$, there exists $k^{\prime} \in \mathbb{Z}_{p^{\ell}}^{*}$ such that $k k^{\prime} \equiv 1\left(\bmod p^{\ell}\right)$. So $K \geq\left\langle a^{p}, b^{p}, c^{p}, b^{j} c^{k}\right\rangle=$ $\left\langle a^{p}, b^{p}, c^{p},\left(b^{j} c^{k}\right)^{k^{\prime}}=b^{j k^{\prime}} c\right\rangle$, and hence $K=\left\langle a^{p}, b^{j k^{\prime}} c, c^{p}\right\rangle \cong \mathbb{Z}_{p^{m-1}} \times \mathbb{Z}_{p^{n}} \times$ $\mathbb{Z}_{p^{\ell-1}}$. 
Lemma 3.6. If $K$ is an index $p^{2}$ subgroup of $\mathbb{Z}_{p^{m}} \times \mathbb{Z}_{p^{n}} \times \mathbb{Z}_{p^{\ell}}$ and $K$ contains the subgroup $\left\langle a^{p}, b^{p}, c^{p}\right\rangle$, then there exist $(p+1)$ index $p$ subgroup of $\mathbb{Z}_{p^{m}} \times$ $\mathbb{Z}_{p^{n}} \times \mathbb{Z}_{p^{\ell}}$ containing $K$.

Proof. Assume that $K$ is a subgroup satisfying the assumption. Then $K$ is one of the $\left(p^{2}+p+1\right)$ subgroups in Lemma 3.4. We only give the proof when $K=\left\langle a^{i} b^{j} c, b^{p}, c^{p}\right\rangle \cong \mathbb{Z}_{p^{m}} \times \mathbb{Z}_{p^{n-1}} \times \mathbb{Z}_{p^{\ell-1}}$ for each integers $i$ and $j$ such that $1 \leq i \leq p-1$ and $0 \leq j \leq p-1$. The remaining can be proved in a similar way. Set $x:=a^{i} b^{j} c, y:=b$ and $z:=c$. Then $\mathbb{Z}_{p^{m}} \times \mathbb{Z}_{p^{n}} \times \mathbb{Z}_{p^{\ell}}=\langle x, y, z\rangle$ and $K=\left\langle x, y^{p}, z^{p}\right\rangle$. Let $H$ be an index $p$ subgroup of $\mathbb{Z}_{p^{m}} \times \mathbb{Z}_{p^{n}} \times \mathbb{Z}_{p^{\ell}}$ containing $K$. Since $K=\left\langle x, y^{p}, z^{p}\right\rangle$ is an index $p^{2}$ subgroup of $\mathbb{Z}_{p^{m}} \times \mathbb{Z}_{p^{n}} \times \mathbb{Z}_{p^{\ell}}$, we have $y^{p k_{1}+i} z^{p k_{2}+j} \in H$ for some integers $k_{1}, k_{2}, i$ and $j$ such that $0 \leq i, j \leq p-1$ and $(i, j) \neq(0,0)$. If $i=0$, then $1 \leq j \leq p-1$ and $H \geq\left\langle x, y^{p}, z^{p}, z^{j}\right\rangle=\left\langle x, y^{p}, z\right\rangle$, and hence $H=\left\langle x, y^{p}, z\right\rangle \cong \mathbb{Z}_{p^{m}} \times \mathbb{Z}_{p^{n-1}} \times \mathbb{Z}_{p^{\ell}}$. Assume now that $i \neq 0$. Then $0 \leq j \leq p-1$ and $H \geq\left\langle x, y^{p}, z^{p}, y^{i} z^{i}\right\rangle$. If $j=0$, then $H \geq\left\langle x, y^{p}, z^{p}, y^{i}\right\rangle=$ $\left\langle x, y, z^{p}\right\rangle$, and hence $H=\left\langle x, y, z^{p}\right\rangle \cong \mathbb{Z}_{p^{m}} \times \mathbb{Z}_{p^{n}} \times \mathbb{Z}_{p^{\ell-1}}$. If $j \neq 0$, then there exists $j^{\prime} \in \mathbb{Z}_{p^{\ell}}^{*}$ such that $j j^{\prime} \equiv 1\left(\bmod p^{\ell}\right)$, and so $H \geq\left\langle x, y^{p}, z^{p}, y^{i j^{\prime}} z\right\rangle=$ $\left\langle x, y^{i j^{\prime}} z, z^{p}\right\rangle$. Hence we have $H=\left\langle x, y^{k} z, z^{p}\right\rangle \cong \mathbb{Z}_{p^{m}} \times \mathbb{Z}_{p^{n}} \times \mathbb{Z}_{p^{\ell-1}}, k=$ $1,2, \ldots, p-1$.

Lemma 3.7. (1) If $m>n>\ell$, then

(8) $b_{m, n, \ell}=b_{m-1, n, \ell}+p\left(b_{m, n-1, \ell}-b_{m-1, n-1, \ell}\right)+p^{2}\left(b_{m, n, \ell-1}-b_{m-1, n, \ell-1}\right)$

$$
-p^{3}\left(b_{m, n-1, \ell-1}-b_{m-1, n-1, \ell-1}\right)+1 \text {. }
$$

(2) If $m=n$ and $n>\ell$, then

(9) $\quad b_{m, m, \ell}=(1+p) b_{m, m-1, \ell}-p b_{m-1, m-1, \ell}$

$$
+p^{2} b_{m, m, \ell-1}-p^{2}(1+p) b_{m, m-1, \ell-1}+p^{3} b_{m-1, m-1, \ell-1}+1 \text {. }
$$

(3) If $m>n$ and $n=\ell$, then

$$
\begin{aligned}
b_{m, n, n}= & b_{m-1, n, n}+p(1+p)\left(b_{m, n, n-1}-b_{m-1, n, n-1}\right) \\
& -p^{3}\left(b_{m, n-1, n-1}-b_{m-1, n-1, n-1}\right)+1 .
\end{aligned}
$$

(4) If $m=n=\ell$, then

$$
\begin{aligned}
b_{m, m, m}= & \left(1+p+p^{2}\right) b_{m, m, m-1}-p\left(1+p+p^{2}\right) b_{m, m-1, m-1} \\
& +p^{3} b_{m-1, m-1, m-1}+1 .
\end{aligned}
$$

Proof. We only give the proof of Eq. (8). The remaining can be proved by a similar way.

By Lemma 3.4 we have

$$
\mathcal{T}\left(\mathbb{Z}_{p^{m}} \times \mathbb{Z}_{p^{n}} \times \mathbb{Z}_{p^{\ell}}\right)=\mathcal{S}\left(\left\langle a, b, c^{p}\right\rangle \cong \mathbb{Z}_{p^{m}} \times \mathbb{Z}_{p^{n}} \times \mathbb{Z}_{p^{\ell-1}}\right)
$$




$$
\begin{aligned}
& \bigcup_{i=1}^{p-1} \mathcal{S}\left(\left\langle a^{i} b, b^{p}, c\right\rangle \cong \mathbb{Z}_{p^{m}} \times \mathbb{Z}_{p^{n-1}} \times \mathbb{Z}_{p^{\ell}}\right) \\
& \bigcup_{1 \leq i, j \leq p-1} \mathcal{S}\left(\left\langle a^{i} b, b^{j} c, c^{p}\right\rangle \cong \mathbb{Z}_{p^{m}} \times \mathbb{Z}_{p^{n}} \times \mathbb{Z}_{p^{\ell-1}}\right) \\
& \bigcup_{i=1}^{p-1} \mathcal{S}\left(\left\langle a^{p}, b, c\right\rangle \cong \mathbb{Z}_{p^{m-1}} \times \mathbb{Z}_{p^{n}} \times \mathbb{Z}_{p^{\ell}}\right) \\
& \bigcup_{\bigcup^{p-1}} \mathcal{S}\left(\left\langle a^{i} c, b, c^{p}\right\rangle \cong \mathbb{Z}_{p^{m}} \times \mathbb{Z}_{p^{n}} \times \mathbb{Z}_{p^{\ell-1}}\right) \\
& \bigcup_{i=1} \mathcal{S}\left(\left\langle a, b^{p}, c\right\rangle \cong \mathbb{Z}_{p^{m}} \times \mathbb{Z}_{p^{n-1}} \times \mathbb{Z}_{p^{\ell}}\right)
\end{aligned}
$$

Using the inclusion-exclusion principle and Lemmas 3.5 and 3.6 we have

$$
\begin{aligned}
& b_{m, n, \ell}-1 \\
& =p^{2} b_{m, n, \ell-1}+p b_{m, n-1, \ell}+b_{m-1, n, \ell} \\
& -\left[p^{2}\left(\begin{array}{c}
p+1 \\
2
\end{array}\right) b_{m, n-1, \ell-1}+p\left(\begin{array}{c}
p+1 \\
2
\end{array}\right) b_{m-1, n, \ell-1}+\left(\begin{array}{c}
p+1 \\
2
\end{array}\right) b_{m-1, n-1, \ell}\right] \\
& +\left[p^{2}\left(\begin{array}{c}
p+1 \\
3
\end{array}\right) b_{m, n-1, \ell-1}+p\left(\begin{array}{c}
p+1 \\
3
\end{array}\right) b_{m-1, n, \ell-1}+\left(\begin{array}{c}
p+1 \\
3
\end{array}\right) b_{m-1, n-1, \ell}\right. \\
& \left.+\left[\left(\begin{array}{c}
p^{2}+p+1 \\
3
\end{array}\right)-\left(p^{2}+p+1\right)\left(\begin{array}{c}
p+1 \\
3
\end{array}\right)\right] b_{m-1, n-1, \ell-1}\right] \\
& -\left[p^{2}\left(\begin{array}{c}
p+1 \\
4
\end{array}\right) b_{m, n-1, \ell-1}+p\left(\begin{array}{c}
p+1 \\
4
\end{array}\right) b_{m-1, n, \ell-1}+\left(\begin{array}{c}
p+1 \\
4
\end{array}\right) b_{m-1, n-1, \ell}\right. \\
& \left.+\left[\left(\begin{array}{c}
p^{2}+p+1 \\
4
\end{array}\right)-\left(p^{2}+p+1\right)\left(\begin{array}{c}
p+1 \\
4
\end{array}\right)\right] b_{m-1, n-1, \ell-1}\right] \\
& +\cdots+(-1)^{p+2}\left[p^{2}\left(\begin{array}{c}
p+1 \\
p+1
\end{array}\right) b_{m, n-1, \ell-1}+p\left(\begin{array}{c}
p+1 \\
p+1
\end{array}\right) b_{m-1, n, \ell-1}\right. \\
& \left.+\left(\begin{array}{c}
p+1 \\
p+1
\end{array}\right) b_{m-1, n-1, \ell}+\left[\left(\begin{array}{c}
p^{2}+p+1 \\
p+1
\end{array}\right)-\left(p^{2}+p+1\right)\left(\begin{array}{c}
p+1 \\
p+1
\end{array}\right)\right] b_{m-1, n-1, \ell-1}\right] \\
& +(-1)^{p+3}\left(\begin{array}{c}
p^{2}+p+1 \\
p+2
\end{array}\right) b_{m-1, n-1, \ell-1}+(-1)^{p+4}\left(\begin{array}{c}
p^{2}+p+1 \\
p+3
\end{array}\right) b_{m-1, n-1, \ell-1} \\
& +\cdots+(-1)^{p^{2}+p+2}\left(\begin{array}{l}
p^{2}+p+1 \\
p^{2}+p+1
\end{array}\right) b_{m-1, n-1, \ell-1} .
\end{aligned}
$$

Thus we have

$$
\begin{aligned}
b_{m, n, \ell}= & b_{m-1, n, \ell}+p b_{m, n-1, \ell}+p^{2} b_{m, n, \ell-1} \\
& -p b_{m-1, n-1, \ell}-p^{2} b_{m-1, n, \ell-1}-p^{3} b_{m, n-1, \ell-1}+p^{3} b_{m-1, n-1, \ell-1}+1 .
\end{aligned}
$$


We prove Eq. (1) by double induction on $n$ and $\ell$. In the following lemma we show that Eq. (1) holds for $\ell=1$.

\section{Lemma 3.8.}

$$
b_{m, n, 1}=(m+n+2)+(m+n) p+\sum_{k=1}^{n} 2(m+n+1-2 k) p^{k+1} .
$$

Proof. We prove Eq. (12) by induction on $n$. Assume first that $n=1$. Note that

$$
\begin{aligned}
b_{m, 1,1}= & b_{m-1,1,1}+p(1+p)\left(b_{m, 1,0}-b_{m-1,1,0}\right) \\
& -p^{3}\left(b_{m, 0,0}-b_{m-1,0,0}\right)+1 \quad \text { by Eq. }(10) \\
= & b_{m-1,1,1}+\left(p+p^{2}\right)(p+1)-p^{3}+1 \quad \text { by Lemma } 3.3 \\
= & b_{m-1,1,1}+2 p^{2}+p+1 .
\end{aligned}
$$

Thus we have

$$
b_{m, 1,1}=b_{1,1,1}+(m-1)\left(2 p^{2}+p+1\right) .
$$

Since

$$
\begin{aligned}
b_{1,1,1} & =\left(1+p+p^{2}\right) b_{1,1,0}-p\left(1+p+p^{2}\right) b_{1,0,0}+p^{3} b_{0,0,0}+1 \quad \text { by Eq. }(11) \\
& =\left(1+p+p^{2}\right)(p+3)-p\left(1+p+p^{2}\right) 2+p^{3}+1 \quad \text { by Lemma } 3.3 \\
& =4+2 p+2 p^{2}
\end{aligned}
$$

we have

$$
b_{m, 1,1}=4+2 p+2 p^{2}+(m-1)\left(2 p^{2}+p+1\right)=m+3+(m+1) p+2 m p^{2} .
$$

Thus Eq. (12) holds for $n=1$.

Assume now that Eq. (12) holds from 1 to $n$ and consider the case for $n+1$.

By Eq. (8) with $(m, n, \ell)=(m, n+1,1)$ we have

$$
\begin{aligned}
b_{m, n+1,1}= & b_{m-1, n+1,1}+p\left(b_{m, n, 1}-b_{m-1, n, 1}\right)+p^{2}\left(b_{m, n+1,0}-b_{m-1, n+1,0}\right) \\
& -p^{3}\left(b_{m, n, 0}-b_{m-1, n, 0}\right)+1 .
\end{aligned}
$$

Note that

$$
\begin{aligned}
b_{m, n, 1}-b_{m-1, n, 1}= & (m+n+2)+(m+n) p+\sum_{k=1}^{n}(2 m+2 n+2-4 k) p^{k+1} \\
& -\left[(m+n+1)+(m+n-1) p+\sum_{k=1}^{n}(2 m+2 n-4 k) p^{k+1}\right] \\
= & 1+p+2 \sum_{k=1}^{n} p^{k+1}
\end{aligned}
$$


by induction hypothesis and

$$
b_{m, n+1,0}-b_{m-1, n+1,0}=\sum_{k=0}^{n+1} p^{k}, \quad b_{m, n, 0}-b_{m-1, n, 0}=\sum_{k=0}^{n} p^{k}
$$

by Lemma 3.3. Thus

$$
\begin{aligned}
b_{m, n+1,1}-b_{m-1, n+1,1} & =1+p+p^{2}+2 \sum_{k=1}^{n} p^{k+2}+\sum_{k=0}^{n+1} p^{k+2}-\sum_{k=0}^{n} p^{k+3} \\
& =1+p+2 p^{2}+2 \sum_{k=1}^{n} p^{k+2}
\end{aligned}
$$

which implies that

$$
b_{m, n+1,1}=b_{n+1, n+1,1}+(m-n-1)\left[1+p+2 p^{2}+2 \sum_{k=1}^{n} p^{k+2}\right] \text {. }
$$

On the other hand, Eq. (9) with $(m, \ell)=(n+1,1)$ gives us that

$$
\begin{aligned}
b_{n+1, n+1,1}= & (1+p) b_{n+1, n, 1}-p b_{n, n, 1}+p^{2} b_{n+1, n+1,0} \\
& -\left(p^{3}+p^{2}\right) b_{n+1, n, 0}+p^{3} b_{n, n, 0}+1 .
\end{aligned}
$$

Since

$$
\begin{aligned}
b_{n+1, n, 1} & =2 n+3+(2 n+1) p+\sum_{k=1}^{n}(4 n+4-4 k) p^{k+1}, \\
b_{n, n, 1} & =2 n+2+2 n p+\sum_{k=1}^{n}(4 n+2-4 k) p^{k+1}
\end{aligned}
$$

by induction hypothesis and

$$
\begin{aligned}
b_{n+1, n+1,0} & =\sum_{k=0}^{n+1}(2 n+3-2 k) p^{k}, \quad b_{n+1, n, 0}=\sum_{k=0}^{n}(2 n+2-2 k) p^{k}, \\
b_{n, n, 0} & =\sum_{k=0}^{n}(2 n+1-2 k) p^{k}
\end{aligned}
$$

by Lemma 3.3, we have

$$
\begin{aligned}
b_{n+1, n+1,1}= & (1+p)\left[2 n+3+(2 n+1) p+\sum_{k=1}^{n}(4 n+4-4 k) p^{k+1}\right] \\
& -p\left[2 n+2+2 n p+\sum_{k=1}^{n}(4 n+2-4 k) p^{k+1}\right] \\
& +p^{2} \sum_{k=0}^{n+1}(2 n+3-2 k) p^{k}
\end{aligned}
$$




$$
\begin{aligned}
& -\left(p^{3}+p^{2}\right) \sum_{k=0}^{n}(2 n+2-2 k) p^{k}+p^{3} \sum_{k=0}^{n}(2 n+1-2 k) p^{k}+1 \\
= & 2 n+4+(2 n+2) p+\sum_{k=1}^{n+1}(4 n+6-4 k) p^{k+1} .
\end{aligned}
$$

Hence, together with Eq. (13) we have

$$
\begin{aligned}
b_{m, n+1,1}= & 2 n+4+(2 n+2) p+\sum_{k=1}^{n+1}(4 n+6-4 k) p^{k+1} \\
& +(m-n-1)\left[1+p+2 p^{2}+2 \sum_{k=1}^{n} p^{k+2}\right] \\
= & (m+n+3)+(m+n+1) p+\sum_{k=1}^{n+1}(4 n+6-4 k) p^{k+1} \\
& +(2 m-2 n-2) p^{2}+2(m-n-1) \sum_{k=1}^{n} p^{k+2} \\
= & (m+n+3)+(m+n+1) p+\sum_{k=1}^{n+1} 2(m+n+2-2 k) p^{k+1} .
\end{aligned}
$$

Therefore, Eq. (12) holds for $n+1$.

Assume now that Eq. (1) holds from 1 to $\ell$ and consider the case for $\ell+1$. Eq. (10) with $(m, n)=(m, \ell+1)$ gives us that

$$
\begin{aligned}
b_{m, \ell+1, \ell+1}= & b_{m-1, \ell+1, \ell+1}+\left(p^{2}+p\right)\left(b_{m, \ell+1, \ell}-b_{m-1, \ell+1, \ell}\right) \\
& -p^{3}\left(b_{m, \ell, \ell}-b_{m-1, \ell, \ell}\right)+1 .
\end{aligned}
$$

By induction hypothesis we know that

$$
\begin{aligned}
& b_{m, \ell+1, \ell}-b_{m-1, \ell+1, \ell} \\
= & \sum_{t=1}^{\ell} t\left[(m+2 \ell-3 t+5) p^{2 t-2}+(m+2 \ell-3 t+3) p^{2 t-1}\right] \\
& +\sum_{k=\ell}^{\ell+1}(\ell+1)(m+\ell+2-2 k) p^{k+\ell}-\sum_{t=1}^{\ell} t\left[(m+2 \ell-3 t+4) p^{2 t-2}\right. \\
& \left.+(m+2 \ell-3 t+2) p^{2 t-1}\right]-\sum_{k=\ell}^{\ell+1}(\ell+1)(m+\ell+1-2 k) p^{k+\ell} \\
= & \sum_{t=1}^{\ell} t\left[p^{2 t-2}+p^{2 t-1}\right]+(\ell+1) p^{2 \ell}+(\ell+1) p^{2 \ell+1}
\end{aligned}
$$


and

$$
\begin{aligned}
b_{m, \ell, \ell}-b_{m-1, \ell, \ell}= & \sum_{t=1}^{\ell} t\left[(m+2 \ell-3 t+4) p^{2 t-2}+(m+2 \ell-3 t+2) p^{2 t-1}\right] \\
& +\sum_{k=\ell}^{\ell}(\ell+1)(m+\ell+1-2 k) p^{k+\ell} \\
& -\sum_{t=1}^{\ell} t\left[(m+2 \ell-3 t+3) p^{2 t-2}+(m+2 \ell-3 t+1) p^{2 t-1}\right] \\
& -\sum_{k=\ell}^{\ell}(\ell+1)(m+\ell-2 k) p^{k+\ell} \\
= & \sum_{t=1}^{\ell} t\left[p^{2 t-2}+p^{2 t-1}\right]+(\ell+1) p^{2 \ell} .
\end{aligned}
$$

Thus we have

$$
\begin{aligned}
& b_{m, \ell+1, \ell+1}-b_{m-1, \ell+1, \ell+1} \\
= & \left(p^{2}+p\right)\left[\sum_{t=1}^{\ell} t\left[p^{2 t-2}+p^{2 t-1}\right]+(\ell+1) p^{2 \ell}+(\ell+1) p^{2 \ell+1}\right] \\
& -p^{3}\left[\sum_{t=1}^{\ell} t\left[p^{2 t-2}+p^{2 t-1}\right]+(\ell+1) p^{2 \ell}\right]+1 \\
= & \sum_{t=1}^{\ell} t\left[p^{2 t-1}+2 p^{2 t}-p^{2 t+2}\right]+2(\ell+1) p^{2 \ell+2}+(\ell+1) p^{2 \ell+1}+1 \\
= & \sum_{t=1}^{\ell+1} t\left[p^{2 t-2}+p^{2 t-1}\right]+(\ell+2) p^{2 \ell+2},
\end{aligned}
$$

which implies that

$$
\begin{aligned}
b_{m, \ell+1, \ell+1}= & b_{\ell+1, \ell+1, \ell+1} \\
& +(m-\ell-1)\left[\sum_{t=1}^{\ell+1} t\left[p^{2 t-2}+p^{2 t-1}\right]+(\ell+2) p^{2 \ell+2}\right] .
\end{aligned}
$$

On the other hand, Eq. (11) with $m=\ell+1$ gives us that

$$
b_{\ell+1, \ell+1, \ell+1}=\left(p^{2}+p+1\right) b_{\ell+1, \ell+1, \ell}-\left(p^{3}+p^{2}+p\right) b_{\ell+1, \ell, \ell}+p^{3} b_{\ell, \ell, \ell}+1 .
$$

Since

$$
b_{\ell+1, \ell+1, \ell}=\sum_{t=1}^{\ell} t\left[(3 \ell-3 t+6) p^{2 t-2}+(3 \ell-3 t+4) p^{2 t-1}\right]
$$




$$
\begin{aligned}
& +\sum_{k=\ell}^{\ell+1}(\ell+1)(2 \ell+3-2 k) p^{k+\ell}, \\
b_{\ell+1, \ell, \ell}= & \sum_{t=1}^{\ell} t\left[(3 \ell-3 t+5) p^{2 t-2}+(3 \ell-3 t+3) p^{2 t-1}\right] \\
& +\sum_{k=\ell}^{\ell}(\ell+1)(2 \ell+2-2 k) p^{k+\ell}
\end{aligned}
$$

and

$$
\begin{aligned}
b_{\ell, \ell, \ell}= & \sum_{t=1}^{\ell} t\left[(3 \ell-3 t+4) p^{2 t-2}+(3 \ell-3 t+2) p^{2 t-1}\right] \\
& +\sum_{k=\ell}^{\ell}(\ell+1)(2 \ell+1-2 k) p^{k+\ell}
\end{aligned}
$$

by induction hypothesis, we have

$$
\begin{aligned}
& b_{\ell+1, \ell+1, \ell+1} \\
& =\left(p^{2}+p+1\right) \\
& \times\left[\sum_{t=1}^{\ell} t\left[(3 \ell-3 t+6) p^{2 t-2}+(3 \ell-3 t+4) p^{2 t-1}\right]\right. \\
& \left.\quad+\sum_{k=\ell}^{\ell+1}(\ell+1)(2 \ell+3-2 k) p^{k+\ell}\right] \\
& -\left(p^{3}+p^{2}+p\right)\left[\sum_{t=1}^{\ell} t\left[(3 \ell-3 t+5) p^{2 t-2}+(3 \ell-3 t+3) p^{2 t-1}\right]\right. \\
& \left.\quad+\sum_{k=\ell}^{\ell}(\ell+1)(2 \ell+2-2 k) p^{k+\ell}\right] \\
& +p^{3}\left[\sum_{t=1}^{\ell} t\left[(3 \ell-3 t+4) p^{2 t-2}+(3 \ell-3 t+2) p^{2 t-1}\right]\right. \\
& \left.\quad+\sum_{k=\ell}^{\ell}(\ell+1)(2 \ell+1-2 k) p^{k+\ell}\right]+1 \\
& =\sum_{t=1}^{\ell} t\left[-p^{2 t+2}+2 p^{2 t}+(3 \ell-3 t+5) p^{2 t-1}+(3 \ell-3 t+6) p^{2 t-2}\right] \\
& +2(\ell+1) p^{2 \ell+2}+2(\ell+1) p^{2 \ell+1}+3(\ell+1) p^{2 \ell}+1
\end{aligned}
$$




$$
=\sum_{t=1}^{\ell+1} t\left[(3 \ell-3 t+7) p^{2 t-2}+(3 \ell-3 t+5) p^{2 t-1}\right]+(\ell+2) p^{2 \ell+2} .
$$

Hence, together with Eq. (14) we have

$$
\begin{aligned}
b_{m, \ell+1, \ell+1}= & \sum_{t=1}^{\ell+1} t\left[(3 \ell-3 t+7) p^{2 t-2}+(3 \ell-3 t+5) p^{2 t-1}\right]+(\ell+2) p^{2 \ell+2} \\
& (m-\ell-1)\left[\sum_{t=1}^{\ell+1} t\left[p^{2 t-2}+p^{2 t-1}\right]+(\ell+2) p^{2 \ell+2}\right] \\
= & \sum_{t=1}^{\ell+1} t\left[(m+2 \ell-3 t+6) p^{2 t-2}+(m+2 \ell-3 t+4) p^{2 t-1}\right] \\
& +\sum_{k=\ell+1}^{\ell+1}(\ell+2)(m+\ell+2-2 k) p^{k+\ell+1} .
\end{aligned}
$$

Therefore, Eq. (1) holds for $n=\ell+1$.

Assume now that Eq. (1) holds from $\ell+1$ to $n$, and consider the case for $n+1$. Eq. (8) with $(m, n, \ell)=(m, n+1, \ell+1)$ gives us that

$$
\begin{aligned}
b_{m, n+1, \ell+1}= & b_{m-1, n+1, \ell+1}+p\left(b_{m, n, \ell+1}-b_{m-1, n, \ell+1}\right) \\
& +p^{2}\left(b_{m, n+1, \ell}-b_{m-1, n+1, \ell}\right)-p^{3}\left(b_{m, n, \ell}-b_{m-1, n, \ell}\right)+1 .
\end{aligned}
$$

By induction hypothesis we know that

$$
\begin{aligned}
& b_{m, n, \ell+1}-b_{m-1, n, \ell+1} \\
= & \sum_{t=1}^{\ell+1} t\left[(m+n+\ell-3 t+5) p^{2 t-2}+(m+n+\ell-3 t+3) p^{2 t-1}\right] \\
& +\sum_{k=\ell+1}^{n}(\ell+2)(m+n+1-2 k) p^{k+\ell+1}-\sum_{t=1}^{\ell+1} t\left[(m+n+\ell-3 t+4) p^{2 t-2}\right. \\
& \left.+(m+n+\ell-3 t+2) p^{2 t-1}\right]-\sum_{k=\ell+1}^{n}(\ell+2)(m+n-2 k) p^{k+\ell+1} \\
= & \sum_{t=1}^{\ell+1} t\left[p^{2 t-2}+p^{2 t-1}\right]+\sum_{k=\ell+1}^{n}(\ell+2) p^{k+\ell+1}, \\
& b_{m, n+1, \ell-b_{m-1, n+1, \ell}}^{\ell} \sum_{t=1}^{\ell} t\left[(m+n+\ell-3 t+5) p^{2 t-2}+(m+n+\ell-3 t+3) p^{2 t-1}\right] \\
& +\sum_{k=\ell}^{n+1}(\ell+1)(m+n+2-2 k) p^{k+\ell}-\sum_{t=1}^{\ell} t\left[(m+n+\ell-3 t+4) p^{2 t-2}\right.
\end{aligned}
$$




$$
\begin{aligned}
& \left.+(m+n+\ell-3 t+2) p^{2 t-1}\right]-\sum_{k=\ell}^{n+1}(\ell+1)(m+n+1-2 k) p^{k+\ell} \\
= & \sum_{t=1}^{\ell} t\left[p^{2 t-2}+p^{2 t-1}\right]+\sum_{k=\ell}^{n+1}(\ell+1) p^{k+\ell}
\end{aligned}
$$

and

$$
\begin{aligned}
& b_{m, n, \ell}-b_{m-1, n, \ell} \\
= & \sum_{t=1}^{\ell} t\left[(m+n+\ell-3 t+4) p^{2 t-2}+(m+n+\ell-3 t+2) p^{2 t-1}\right] \\
& +\sum_{k=\ell}^{n}(\ell+1)(m+n+1-2 k) p^{k+\ell}-\sum_{t=1}^{\ell} t\left[(m+n+\ell-3 t+3) p^{2 t-2}\right. \\
& \left.+(m+n+\ell-3 t+1) p^{2 t-1}\right]-\sum_{k=\ell}^{n}(\ell+1)(m+n-2 k) p^{k+\ell} \\
= & \sum_{t=1}^{\ell} t\left[p^{2 t-2}+p^{2 t-1}\right]+\sum_{k=\ell}^{n}(\ell+1) p^{k+\ell} .
\end{aligned}
$$

Hence we have

$$
\begin{aligned}
& b_{m, n+1, \ell+1}-b_{m-1, n+1, \ell+1} \\
= & p\left[\sum_{t=1}^{\ell+1} t\left[p^{2 t-2}+p^{2 t-1}\right]+\sum_{k=\ell+1}^{n}(\ell+2) p^{k+\ell+1}\right] \\
& +p^{2}\left[\sum_{t=1}^{\ell} t\left[p^{2 t-2}+p^{2 t-1}\right]+\sum_{k=\ell}^{n+1}(\ell+1) p^{k+\ell}\right] \\
& -p^{3}\left[\sum_{t=1}^{\ell} t\left[p^{2 t-2}+p^{2 t-1}\right]+\sum_{k=\ell}^{n}(\ell+1) p^{k+\ell}\right]+1 \\
= & \sum_{t=1}^{\ell+1} t\left[p^{2 t-2}+p^{2 t-1}\right]+\sum_{k=\ell+1}^{n+1}(\ell+2) p^{k+\ell+1},
\end{aligned}
$$

which implies that

$$
\begin{aligned}
& b_{m, n+1, \ell+1} \\
= & b_{n+1, n+1, \ell+1} \\
& +(m-n-1)\left[\sum_{t=1}^{\ell+1} t\left[p^{2 t-2}+p^{2 t-1}\right]+\sum_{k=\ell+1}^{n+1}(\ell+2) p^{k+\ell+1}\right] .
\end{aligned}
$$


On the other hand, by Eq. $(9)$ with $(m, \ell)=(n+1, \ell+1)$ we have

$$
\begin{aligned}
b_{n+1, n+1, \ell+1}= & (p+1) b_{n+1, n, \ell+1}-p b_{n, n, \ell+1}+p^{2} b_{n+1, n+1, \ell} \\
& -\left(p^{3}+p^{2}\right) b_{n+1, n, \ell}+p^{3} b_{n, n, \ell}+1 .
\end{aligned}
$$

Since

$$
\begin{aligned}
& b_{n+1, n, \ell+1}=\sum_{t=1}^{\ell+1} t\left[(2 n+\ell-3 t+6) p^{2 t-2}+(2 n+\ell-3 t+4) p^{2 t-1}\right] \\
& +\sum_{k=\ell+1}^{n}(\ell+2)(2 n+2-2 k) p^{k+\ell+1} \\
& b_{n, n, \ell+1}=\sum_{t=1}^{\ell+1} t\left[(2 n+\ell-3 t+5) p^{2 t-2}+(2 n+\ell-3 t+3) p^{2 t-1}\right] \\
& +\sum_{k=\ell+1}^{n}(\ell+2)(2 n+1-2 k) p^{k+\ell+1}, \\
& b_{n+1, n+1, \ell}=\sum_{t=1}^{\ell} t\left[(2 n+\ell-3 t+6) p^{2 t-2}+(2 n+\ell-3 t+4) p^{2 t-1}\right] \\
& +\sum_{k=\ell}^{n+1}(\ell+1)(2 n+3-2 k) p^{k+\ell} \\
& b_{n+1, n, \ell}=\sum_{t=1}^{\ell} t\left[(2 n+\ell-3 t+5) p^{2 t-2}+(2 n+\ell-3 t+3) p^{2 t-1}\right] \\
& +\sum_{k=\ell}^{n}(\ell+1)(2 n+2-2 k) p^{k+\ell}
\end{aligned}
$$

and

$$
\begin{aligned}
b_{n, n, \ell}= & \sum_{t=1}^{\ell} t\left[(2 n+\ell-3 t+4) p^{2 t-2}+(2 n+\ell-3 t+2) p^{2 t-1}\right] \\
& +\sum_{k=\ell}^{n}(\ell+1)(2 n+1-2 k) p^{k+\ell}
\end{aligned}
$$

by induction hypothesis, we have

$$
\begin{aligned}
& b_{n+1, n+1, \ell+1} \\
= & (p+1)\left[\sum_{t=1}^{\ell+1} t\left[(2 n+\ell-3 t+6) p^{2 t-2}+(2 n+\ell-3 t+4) p^{2 t-1}\right]\right.
\end{aligned}
$$




$$
\begin{aligned}
& \left.+\sum_{k=\ell+1}^{n}(\ell+2)(2 n+2-2 k) p^{k+\ell+1}\right]-p\left[\sum _ { t = 1 } ^ { \ell + 1 } t \left[(2 n+\ell-3 t+5) p^{2 t-2}\right.\right. \\
& \left.\left.+(2 n+\ell-3 t+3) p^{2 t-1}\right]+\sum_{k=\ell+1}^{n}(\ell+2)(2 n+1-2 k) p^{k+\ell+1}\right] \\
& +p^{2}\left[\sum_{t=1}^{\ell} t\left[(2 n+\ell-3 t+6) p^{2 t-2}+(2 n+\ell-3 t+4) p^{2 t-1}\right]\right. \\
& \left.+\sum_{k=\ell}^{n+1}(\ell+1)(2 n+3-2 k) p^{k+\ell}\right]-\left(p^{3}+p^{2}\right)\left[\sum _ { t = 1 } ^ { \ell } t \left[(2 n+\ell-3 t+5) p^{2 t-2}\right.\right. \\
& \left.\left.+(2 n+\ell-3 t+3) p^{2 t-1}\right]+\sum_{k=\ell}^{n}(\ell+1)(2 n+2-2 k) p^{k+\ell}\right] \\
& +p^{3}\left[\sum_{t=1}^{\ell} t\left[(2 n+\ell-3 t+4) p^{2 t-2}+(2 n+\ell-3 t+2) p^{2 t-1}\right]\right. \\
& \left.+\sum_{k=\ell}^{n}(\ell+1)(2 n+1-2 k) p^{k+\ell}\right]+1 \\
= & \sum_{t=1}^{\ell+1} t\left[(2 n+\ell-3 t+6) p^{2 t-2}+(2 n+\ell-3 t+5) p^{2 t-1}+p^{2 t}\right] \\
& +\sum_{t=1}^{\ell} t\left[p^{2 t}-p^{2 t+2}\right]+\sum_{k=\ell+1}^{n}(\ell+2)(2 n+2-2 k) p^{k+\ell+1} \\
& +\sum_{k=\ell+1}^{n}(\ell+2) p^{k+\ell+2}+\sum_{k=\ell}^{n+1}(\ell+1)(2 n+3-2 k) p^{k+\ell+2} \\
& -\sum_{k=\ell}^{n}(\ell+1)(2 n+2-2 k) p^{k+\ell+2}-\sum_{k=\ell}^{n}(\ell+1) p^{k+\ell+3}+1 \\
& \sum_{t=1}^{\ell+1} t\left[(2 n+\ell-3 t+7) p^{2 t-2}+(2 n+\ell-3 t+5) p^{2 t-1}\right] \\
& \sum_{k=\ell+1}^{n+1}(\ell+2)(2 n+3-2 k) p^{k+\ell+1} \cdot
\end{aligned}
$$

Hence, together with Eq. (15) we have

$$
b_{m, n+1, \ell+1}=\sum_{t=1}^{\ell+1} t\left[(2 n+\ell-3 t+7) p^{2 t-2}+(2 n+\ell-3 t+5) p^{2 t-1}\right]
$$




$$
\begin{aligned}
& +\sum_{k=\ell+1}^{n+1}(\ell+2)(2 n+3-2 k) p^{k+\ell+1} \\
& +(m-n-1)\left[\sum_{t=1}^{\ell+1} t\left[p^{2 t-2}+p^{2 t-1}\right]+\sum_{k=\ell+1}^{n+1}(\ell+2) p^{k+\ell+1}\right] \\
& =\sum_{t=1}^{\ell+1} t\left[(m+n+\ell-3 t+6) p^{2 t-2}+(m+n+\ell-3 t+4) p^{2 t-1}\right] \\
& +\sum_{k=\ell+1}^{n+1}(\ell+2)(m+n+2-2 k) p^{k+\ell+1} .
\end{aligned}
$$

Therefore, Eq. (1) holds for $n+1$. Consequently, we have proved Theorem 2.1.

In general, for the group $\mathbb{Z}_{p^{k_{1}}} \times \mathbb{Z}_{p^{k_{2}}} \times \cdots \times \mathbb{Z}_{p^{k_{\ell}}}$, where $k_{1}, k_{2}, \ldots, k_{\ell}$ are positive integers, $\ell$ is a positive integer such that $\ell \geq 4$ and $p$ is a prime number, it seems not easy to obtain an explicit formula for the total number of subgroups with the method used in this paper.

\section{References}

[1] G. Călugăreanu, The total number of subgroups of a finite abelian group, Sci. Math. Jpn. 60 (2004), no. 1, 157-167.

[2] I. J. Davies, Enumeration of certain subgroups of abelian p-groups, Proc. Edinburgh Math. Soc. 13 (1962), no. 2, 1-4.

[3] S. Delsarte, Fonctions de Möbius sur les groupes abéliens finis, Ann. of Math. 49 (1948), no. 2, 600-609.

[4] P. Dyubyuk, On the number of subgroups of a finite abelian group, Soviet Math. 2 (1961), 298-300.

[5] J. Petrillo, Counting subgroups in a direct product of finite cyclic groups, College Math. J. 42 (2011), no. 3, 215-222.

[6] Y. Yeh, On prime power abelian groups, Bull. Amer. Math. Soc. 54 (1948), 323-327.

Department of Mathematics

Gangneung-Wonju National University

GANGNEUng 210-702, Korea

E-mail address: jumokoh@gwnu.ac.kr 